\title{
MC-ICP-MS铀同位素的高精度法拉第杯静态分析
}

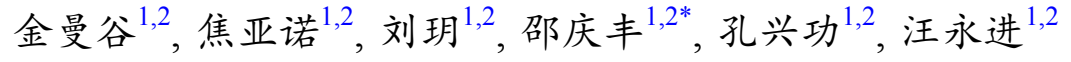 \\ 1. 南京师范大学地理科学学院, 虚拟地理环境教育部重点实验室, 南京 210023; \\ 2. 江苏省地理信息资源开发与利用协同创新中心, 南京 210023 \\ *联系人, E-mail: qingfengshao@njnu.edu.cn \\ 2021-11-06 收稿, 2022-02-03 修回, 2022-02-05 接受, 2022-02-09 网络版发表 \\ 国家自然科学基金(41877430)资助
}

\begin{abstract}
摘要 铀同位素是地球和环境等科学领域的重要研究工具, 然而铀同位素极大的丰度差异一直是高精度测试分析 的难题. 近年来质谱分析仪器公司推出的 $10^{13} \Omega$ 高阻信号放大器显著提高了信噪比, 适用于测试低丰度的 ${ }^{234} \mathrm{U}$. 组 合使用 $10^{11} 、 10^{12}$ 和 $10^{13} \Omega$ 的高阻信号放大器可实现多接收器电感耦合等离子体质谱(Multiple Collector Inductively Coupled Plasma Mass Spectrometry, MC-ICP-MS)的铀同位素静态分析, 即用 $10^{13} \Omega$ 高阻放大器的法拉第杯接收 ${ }^{234} \mathrm{U}$, $10^{12} \Omega$ 的法拉第杯接收 ${ }^{233} \mathrm{U}$ 和 ${ }^{236} \mathrm{U}$, 以及 $10^{11} \Omega$ 的法拉第杯接收 ${ }^{235} \mathrm{U}$ 和 ${ }^{238} \mathrm{U}$. MC-ICP-MS静态测试 ${ }^{234} \mathrm{U} /{ }^{238} \mathrm{U}$ 和 ${ }^{238} \mathrm{U} /{ }^{235} \mathrm{U}$ 比值的主要校正因子包括法拉第杯的基线、放大器相对增益、仪器本底、 ${ }^{238} \mathrm{U}$ 拖尾、铀氢化物干扰以及质量歧视 等. 组合使用这3种放大器多次重复测试了国际标准物质HU-1和SRM950a, 以及国内标准物质 GBW04412和 GBW04428的铀同位素组成. 新方法的测试结果与国内外已发表的测试数据高度一致, 验证了新方法的可靠性. 实 验表明, 新的MC-ICP-MS 铀同位素法拉第杯静态分析方法高效便捷, 可以对 $20 \sim 30 \mathrm{ng}$ 铀同步分析 ${ }^{234} \mathrm{U} /{ }^{238} \mathrm{U}$ 和 ${ }^{238} \mathrm{U} /{ }^{235} \mathrm{U}$, 精度可分别达到 $0.3 \%{ }^{\circ} \sim 1 \%$ 和 $0.3 \varepsilon \sim 0.7 \varepsilon( \pm 2 \sigma)$, 用时约为 $20 \mathrm{~min}$. 此外, 在样品测试时可以根据样品的铀含 量以及对分析精度的要求灵活调整MC-ICP-MS检测系统的高阻信号放大器的排列方式, 因此新方法可以广泛用于 地质样品的铀同位素分析.
\end{abstract}

关键词多接收器电感耦合等离子体质谱, $10^{13} \Omega$ 高阻信号放大器, 铀标准物质, 铀同位素

天然铀同位素 ${ }^{238} \mathrm{U} 、{ }^{235} \mathrm{U}$ 和 ${ }^{234} \mathrm{U}$ 的相对丰度分别为 $\sim 99.274 \% 、 \sim 0.720 \%$ 和 $\sim 0.0054 \%{ }^{[1]}$, 其中 ${ }^{238} \mathrm{U}$ 和 ${ }^{235} \mathrm{U}$ 是 两个长寿命的放射性母体核素，半衰期分别为 45 亿年 和 7 亿年 ${ }^{[2]}$. 自 20 世纪70年代以来, ${ }^{238} \mathrm{U} /{ }^{235} \mathrm{U}$ 原子比值 长期被认为是常数 $137.88^{[3]}$, 并被广泛用于 $\mathrm{U}-\mathrm{Pb}$ 和 ${ }^{230} \mathrm{Th} / \mathrm{U}$ 测年以及校正同位素质谱分析的质量歧视 (mass bias) 效应 ${ }^{[4]}$. 近年来, 随着分析测试技术不断改 进, 越来越多的研究表明自然界含铀物质的 ${ }^{238} U{ }^{235} U$ 比 值存在显著变化, 并不恒定. 例如, Condon等人 ${ }^{[5]}$ 利用 IRMM-3636双稀释剂 ${ }^{233} U$ 和 $\left.{ }^{236} U\right)$ 分析了常用的天然铀
标准物质CRM-112a、SRM950a和HU-1 的 ${ }^{238} U /{ }^{235} \mathrm{U}$ 比 值，其结果与 137.88 的差异高达 $0.8 \%$. 又如，Hiess等 人 ${ }^{[6]}$ 分析了低温和高温条件下形成的多种地质样品(海 水、方解石、锆石等), 发现 ${ }^{238} \mathrm{U} /{ }^{235} \mathrm{U}$ 变化范围达 到 $\sim 5 \%$.

核素 ${ }^{234} \mathrm{U}$ 是 ${ }^{238} \mathrm{U}$ 衰变链中半衰期最长的中间子体, 半衰期约为 24.6 万年. 在长期平衡状态下, ${ }^{234} \mathrm{U} /{ }^{238} \mathrm{U}$ 原 子比值为 $54.97 \times 10^{-6[7]}$. 由于衰变链中各元素地球化学 性质的差异(如溶解性和吸附性), 矿物在风化、侵蚀、 搬运和沉积等过程中容易发生铀系衰变不平衡. 受到 
$\alpha$-粒子的反冲作用 $(\alpha$-recoil $),{ }^{234} \mathrm{U}$ 比 ${ }^{238} \mathrm{U}$ 更易于从矿物 中淋失, 因此地表水中 ${ }^{234} \mathrm{U} /{ }^{238} \mathrm{U}$ 活度比通常大于1. 河

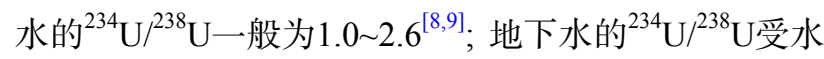
流速率和围岩性质等因素影响，一般为 $1 \sim 4^{[10]}$. 海水 的 ${ }^{234} \mathrm{U} /{ }^{238} \mathrm{U}$ 约为 $1.148^{[11,12]}$, 全球范围的变化小于 $2.5 \%{ }^{[9]}$. 水环境中生成的次生碳酸盐(如珊瑚、钟乳石 等)通常含有微量铀并且初始 ${ }^{234} \mathrm{U} /{ }^{238} \mathrm{U}>1$, 是 ${ }^{230} \mathrm{Th} / \mathrm{U}$ 和 $\mathrm{U}-\mathrm{Pb}$ 测年的理想材料 ${ }^{[7,13,14]}$. 由于 ${ }^{234} \mathrm{U}$ 的优先淋失, 陆 源碎屑沉积物中微米级的颗粒 ${ }^{234} \mathrm{U} /{ }^{238} \mathrm{U}$ 通常小于 1 . 这 种现象近年来被用于研究沉积物从基岩分离到暴露搬 运再到堆积所经过的时间，即破碎年龄 ${ }^{[15,16]}$. 除地质年 代学之外, 铀同位素在地球和环境科学等诸多领域都 有重要应用 ${ }^{[17,18]}$.

自20世纪80年代后期, 热电离质谱(Thermal Ionization Mass Spectrometry, TIMS)代替 $\alpha$-能谱, 成为铀钍同 位素分析测试的主要技术. TIMS技术将铀钍同位素的 分析精度提高了一个数量级，达到 $3 \% 0 \sim 5 \%{ }^{[13]}$. 20 世纪 90 年代后期以来，多接收器电感耦合等离子体质谱仪 (Multiple Collector Inductively Coupled Plasma Mass Spectrometry, MC-ICP-MS)逐步用于铀针同位素分 析 $^{[19]}$. MC-ICP-MS的铀针电离效率 $>95 \%$, 有助于进一 步提高测试精度 ${ }^{[4]}$. 目前, MC-ICP-MS的 ${ }^{234} \mathrm{U} /{ }^{238} \mathrm{U}$ 分析 精度常规可达到 $1 \% 0 \sim 2 \%$, 最佳可优于 $1 \%{ }^{[7,20]} ;{ }^{238} U{ }^{235} U$ 的测量精度则可达到 $10^{-5}$ 水平 ${ }^{[6,7,21 ~ 23]}$.

铀同位素质谱分析的难点在于 ${ }^{234} \mathrm{U}$ 与 ${ }^{238} \mathrm{U}$ 的丰度 相差4 5个数量级, 这使得不能用标准高阻信号放大器 $\left(10^{11} \Omega\right)$ 高精度分析 ${ }^{234} \mathrm{U} /{ }^{238} \mathrm{U}$. 目前MC-ICP-MS的铀同 位素分析方法主要有3种: (1) 跳峰测试法. 该方法用离 子计数器依次测量 ${ }^{233} \mathrm{U} 、{ }^{234} \mathrm{U} 、{ }^{235} \mathrm{U}$ 和 ${ }^{236} \mathrm{U}$ 离子束的信 号强度, 其中 ${ }^{233} \mathrm{U}$ 和 ${ }^{236} \mathrm{U}$ 为稀释剂. 将 ${ }^{238} \mathrm{U} /{ }^{235} \mathrm{U}$ 视为定值, 通过测量 ${ }^{234} U{ }^{235} U$ 进而计算 ${ }^{234} U{ }^{238} U^{[24]}$. (2) 法拉第杯与 离子计数器组合测试法. 该方法用法拉第杯测量丰度 较大的 ${ }^{238} \mathrm{U}$ 和 ${ }^{235} \mathrm{U}$, 同时用离子计数器测量 ${ }^{234} \mathrm{U}$. 在测量 未知样品的前后分别测量标准样品, 根据标准样品 的 ${ }^{234} \mathrm{U} /{ }^{238} \mathrm{U}$ 测量值与真值比较而校正检测器之间的相 对增益 ${ }^{[20,25,26]}$. 此外, 用这两种离子检测器交替测试离 子束强度约为 $5 \mathrm{mV}$ 的 ${ }^{235} \mathrm{U}$ 或 ${ }^{236} \mathrm{U}$ 也可以计算相对增

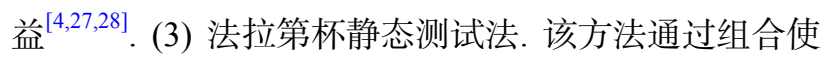
用不同量级的高阻信号放大器实现铀同位素的静态测 试. 如Andersen等人 ${ }^{[20]}$ 分别用 $10^{9} \Omega$ 的法拉第杯测 量 ${ }^{238} \mathrm{U}$, 用 $10^{11} \Omega$ 的法拉第杯测量 ${ }^{235} \mathrm{U} 、{ }^{234} \mathrm{U}$ 和稀释剂, 使得 ${ }^{234} \mathrm{U} /{ }^{238} \mathrm{U}$ 测量精度达到 $0.3 \%$. 又如, Cheng等人 ${ }^{[7]}$
分别用 $10^{10} \Omega$ 的法拉第杯测量 ${ }^{238} \mathrm{U}$ ，用 $10^{11} \Omega$ 的法拉第 杯测量其他铀同位素，使得 ${ }^{234} \mathrm{U} /{ }^{238} \mathrm{U}$ 测量精度高达 $0.1 \%$ $~ 0.3 \%$. 然而, $10^{11} \Omega$ 放大器的信噪比相对较低, ${ }^{234} \mathrm{U}$ 离子束强度需 $>30 \mathrm{mV}$ 才能实现高精度测量, 因此 用 $10^{11} \Omega$ 的法拉第杯高精度测量 ${ }^{234} \mathrm{U}$ 至少需要消耗 $400 \sim 1000 \mathrm{ng}$ 铀 $^{[7,20]}$.

2014年, Thermo Fisher Scientific公司推出用于测 量弱离子束信号的 $10^{13} \Omega$ 高电阻放大器. 测试表明与标 准的 $10^{11} \Omega$ 信号放大器相比, $10^{13} \Omega$ 的信噪比提高了 5 倍 以上; 对于强度 $>20000 \mathrm{cps}$ 的离子束, 用 $10^{13} \Omega$ 的法拉第 杯可以取得比离子计数器更高的测试精度 ${ }^{[29,30]} \cdot 10^{13} \Omega$ 电阻放大器为高精度测量 ${ }^{234} \mathrm{U}$ 提供了新方案, 但目前在 国内外尚未得到广泛使用. 为此, 我们尝试组合使用 $10^{11} 、 10^{12}$ 和 $10^{13} \Omega$ 高阻放大器对铀同位素静态分析的 新方法, 即同时用 $10^{13} \Omega$ 的法拉第杯测试 ${ }^{234} \mathrm{U}, 10^{12} \Omega$ 的 法拉第杯测量 ${ }^{233} \mathrm{U}$ 和 ${ }^{236} \mathrm{U}$ ，以及 $10^{11} \Omega$ 的法拉第杯测 量 ${ }^{235} \mathrm{U}$ 和 ${ }^{238} \mathrm{U}$. 此研究目的在于实现 ${ }^{234} \mathrm{U} /{ }^{238} \mathrm{U}$ 和 ${ }^{238} \mathrm{U} /{ }^{235} \mathrm{U}$ 的高精度静态测试并且相对减少样品用量. 用新的测 量方法, 我们多次重复分析国际标准物质 HU-1 和 SRM950a, 以及国内标准物质GBW04428和GBW04412 的铀同位素组成并与国内外已发表的数据对比，验证 新方法的可靠性.

\section{1 实验条件}

我们的铀同位素分析在Thermo Fisher Neptune MC-ICP-MS仪器上进行, 该仪器安装于南京师范大学 石笋同位素实验室. 目前仪器配备1个二次电子倍增器 (secondary electron multiplier, SEM)、9个法拉第杯和4 种高阻信号放大器, 放大器分别为 $10^{13} \Omega(A 4) 、 10^{12} \Omega$ $(\mathrm{A} 6$ 和 $\mathrm{A} 8) 、 10^{11} \Omega(\mathrm{A} 1 、 \mathrm{~A} 2 、 \mathrm{~A} 3 、 \mathrm{~A} 5 、 \mathrm{~A} 7$ 和 $\mathrm{A} 9)$ 和 $10^{10} \Omega(\mathrm{A} 10)$. 仪器进样端口由Jet-样品雉、X-截取雉和 PFEIFFER干泵 $\left(100 \mathrm{~m}^{3} / \mathrm{h}\right)$ 组成. 我们使用自动进样器 Cetac ASX-520和膜去溶雾化系统Aridus II进样, 溶液 提取速率约为 $50 \mu \mathrm{L} / \mathrm{min}$, 介质为 $0.5 \mathrm{~mol} / \mathrm{L}^{-} \mathrm{HNO}_{3}+0.01$ $\mathrm{mol} / \mathrm{L} \mathrm{HF}$ 混合酸. 表 1 为仪器的常用工作参数, 通常铀 $\left({ }^{238} \mathrm{U}\right)$ 的灵敏度为 $750 \sim 1000 \mathrm{~V} / \mathrm{ppm}(1 \mathrm{ppm}=1 \mu \mathrm{g} / \mathrm{g})$.

我们使用的铀同位素标准样品包括国际标准物质 HU - 1 和 SRM950a、国内标准物质 GBW04412 和 GBW04428. 稀释剂为IRMM-3636, 其 ${ }^{233} \mathrm{U} /{ }^{236} \mathrm{U}=$ $1.01906(16) 、{ }^{234} \mathrm{U} /{ }^{236} \mathrm{U}=0.00036606(48) 、{ }^{235} \mathrm{U} /{ }^{236} \mathrm{U}=$ $0.000045480(74) 、{ }^{238} \mathrm{U} /{ }^{236} \mathrm{U}=0.00023481(38)^{[31]}$. 超纯水 由美国Millipore纯水系统所制, 优级纯盐酸和硝酸经 
表 1 Neptune MC-ICP-MS仪器工作参数

Table 1 Neptune MC-ICP-MS instrument parameters used for measurements

\begin{tabular}{cc}
\hline 工作参数 & 数值 \\
\hline 射频功率 & $1200 \mathrm{~W}$ \\
提取电压 & $-2000 \mathrm{~V}$ \\
质量分辨率 & 低 \\
雾化室温度 & $110^{\circ} \mathrm{C}$ \\
膜室温度 & $160^{\circ} \mathrm{C}$ \\
截取雉 & $\mathrm{X}$ \\
样品雉 & $\mathrm{Jet}$ \\
吹扫气(Ar) $(\mathrm{Ar}$ & $3 \sim 5 \mathrm{~L} / \mathrm{min}$ \\
增敏气( $\left.\mathrm{N}_{2}\right)$ & $3 \sim 10 \mathrm{~mL} / \mathrm{min}$ \\
雾化器 & $\sim 50 \mu \mathrm{L} / \mathrm{min}$ \\
样品气(Ar) & $0.6 \sim 0.8 \mathrm{~L} / \mathrm{min}$ \\
\hline
\end{tabular}

Savillex DST-1000系统两次亚沸腾蒸馏后使用. Trisken UTEVA离子交换树脂用于分离和纯化样品中的铀元 素. 所有的化学分析流程都在超净实验室的通风梪内 完成, 化学流程空白为 $7 \mathrm{pg}{ }^{238} \mathrm{U}$ 和 $\sim 0.8 \mathrm{fg}{ }^{234} \mathrm{U}$.

\section{2 铀同位素的测试和数据校正}

\subsection{MC-ICP-MS的杯配置和数据采集}

表2展示了采用的杯配置和数据采集方式: ${ }^{233} \mathrm{U}$ 、 ${ }^{234} \mathrm{U} 、{ }^{235} \mathrm{U} 、{ }^{236} \mathrm{U} 、{ }^{238} \mathrm{U}$ 分别用L1、C、H1、H2、H3位 置的法拉第杯同步测量，信号放大器分别为 $10^{12}$ 、 $10^{13} 、 10^{11} 、 10^{12} 、 10^{11} \Omega$. 图1为中心杯扫描 ${ }^{234} \mathrm{U}$ 时铀 同位素呈现的峰形.

在测量样品之前, 先用 $1 \mathrm{~mol} / \mathrm{L} \mathrm{HNO}_{3}+0.01 \mathrm{~mol} / \mathrm{L}$
HF混合酸清洗进样系统 3 min; 之后测量各个法拉第 杯的基线 $6 \mathrm{~min}$; 随后引入 $0.5 \mathrm{~mol} / \mathrm{L} \mathrm{HNO}_{3}+0.01 \mathrm{~mol} / \mathrm{L}$ $\mathrm{HF}$ 混合酸，再用SEM扫描各个铀同位素的本底 $4 \mathrm{~min}$. 样品测试溶液为 $\sim 0.5 \mathrm{~mL}$, 信号强度 ${ }^{238} \mathrm{U}$ 为 $30 \sim 40 \mathrm{~V},{ }^{234} \mathrm{U}$ 为 $1 \sim 5 \mathrm{mV},{ }^{233} \mathrm{U}$ 和 ${ }^{236} \mathrm{U}$ 为 $50 \sim 250 \mathrm{mV}$ (相对于 $10^{11} \Omega$ 信号 放大器), 信号积分时间为 $8.389 \mathrm{~s}$. 该方法测量一个样品 耗时约为 $20 \mathrm{~min}$, 包括清洗仪器流程.

\section{2 增益校正}

我们的铀同位素静态测量方法使用了 $10^{11} 、 10^{12}$ 和 $10^{13} \Omega$ 高阻信号放大器，而所用的MC-ICP-MS目前只 限于自动校正 $10^{12} 、 10^{11}$ 和 $10^{10} \Omega$ 的法拉第杯增益. 为 校正 $10^{13} \Omega$ 的法拉第杯的增益, 先用仪器自动校正 $10^{11}$ 和 $10^{12} \Omega$ 放大器增益; 之后引人铀的溶液 ${ }^{238} \mathrm{U}$ 约为 $30 \mathrm{~V})$, 在 $\mathrm{H} 1$ 位置用 $10^{13}$ 和 $10^{11} \Omega$ 法拉第杯两次测 量 ${ }^{235} \mathrm{U}$ ，每次都在 $\mathrm{H} 3$ 的位置用 $10^{11} \Omega$ 的法拉第杯测 量 ${ }^{238} \mathrm{U}$. 将前后两次测量的 ${ }^{238} \mathrm{U} /{ }^{235} \mathrm{U}$ 值之比视为 $10^{13} \Omega$ 放大器的相对增益 $\left({ }^{238} \mathrm{U}_{11}{ }^{235} \mathrm{U}_{13}\right) /\left({ }^{238} \mathrm{U}_{11} /{ }^{235} \mathrm{U}_{11}\right)$. 图2展 示了 2021 年6 10月，本实验室 $10^{13} \Omega$ 放大器增益的波 动, 平均值约为 0.0103679 .

\section{3 基线和本底校正}

在每次分析样品之前检测法拉第杯的基线. 图3展 示了 2021 年8 9月间在L1、C、H1、H2和H3位置的法 拉第杯的基线 $(n=153)$. 这些位置的法拉第杯分别连接 信号放大器A6 $\left(10^{12} \Omega\right) 、 A 4\left(10^{13} \Omega\right) 、 A 5\left(10^{11} \Omega\right) 、 A 8$ $\left(10^{12} \Omega\right)$ 和 $A 7\left(10^{11} \Omega\right)$, 用于测量 ${ }^{233} U 、{ }^{234} U 、{ }^{235} U 、{ }^{236} U$ 和 ${ }^{238} \mathrm{U}$ 离子束. 数据显示这些法拉第杯的基线呈现长期 稳定的趋势，平均值分别为 $1.194 、-0.659 、-1.231$ 、

表 2 Neptune MC-ICP-MS铀同位素分析的杯结构和数据采集方式

Table 2 Neptune MC-ICP-MS cup configuration and data acquisition for U isotopic measurement

\begin{tabular}{|c|c|c|c|c|c|c|c|c|c|}
\hline 探测器 & L4 & L3 & $\mathrm{L} 2$ & L1 & $\mathrm{C}$ & H1 & $\mathrm{H} 2$ & $\mathrm{H} 3$ & $\mathrm{H} 4$ \\
\hline 放大器 $(\Omega)$ & $10^{11}$ & $10^{11}$ & $10^{11}$ & $10^{12}$ & SEM & $10^{11}$ & $10^{12}$ & $10^{11}$ & $10^{11}$ \\
\hline \multirow{5}{*}{$\begin{array}{c}\text { 仪器本底 } \\
\text { (baseline at start) }\end{array}$} & & & & & ${ }^{233} \mathrm{U}$ & & & & \\
\hline & & & & & ${ }^{234} \mathrm{U}$ & & & & \\
\hline & & & & & ${ }^{235} \mathrm{U}$ & & & & \\
\hline & & & & & ${ }^{236} \mathrm{U}$ & & & & \\
\hline & & & & & ${ }^{238} \mathrm{U}$ & & & & \\
\hline 探测器 & L4 & L3 & L2 & $\mathrm{L} 1$ & $\mathrm{C}$ & H1 & $\mathrm{H} 2$ & $\mathrm{H} 3$ & $\mathrm{H} 4$ \\
\hline 放大器 $(\Omega)$ & $10^{11}$ & $10^{11}$ & $10^{11}$ & $10^{12}$ & $10^{13}$ & $10^{11}$ & $10^{12}$ & $10^{11}$ & $10^{11}$ \\
\hline 样品铀 & & & & ${ }^{233} \mathrm{U}$ & ${ }^{234} \mathrm{U}$ & ${ }^{235} \mathrm{U}$ & ${ }^{236} \mathrm{U}$ & ${ }^{238} \mathrm{U}$ & \\
\hline
\end{tabular}




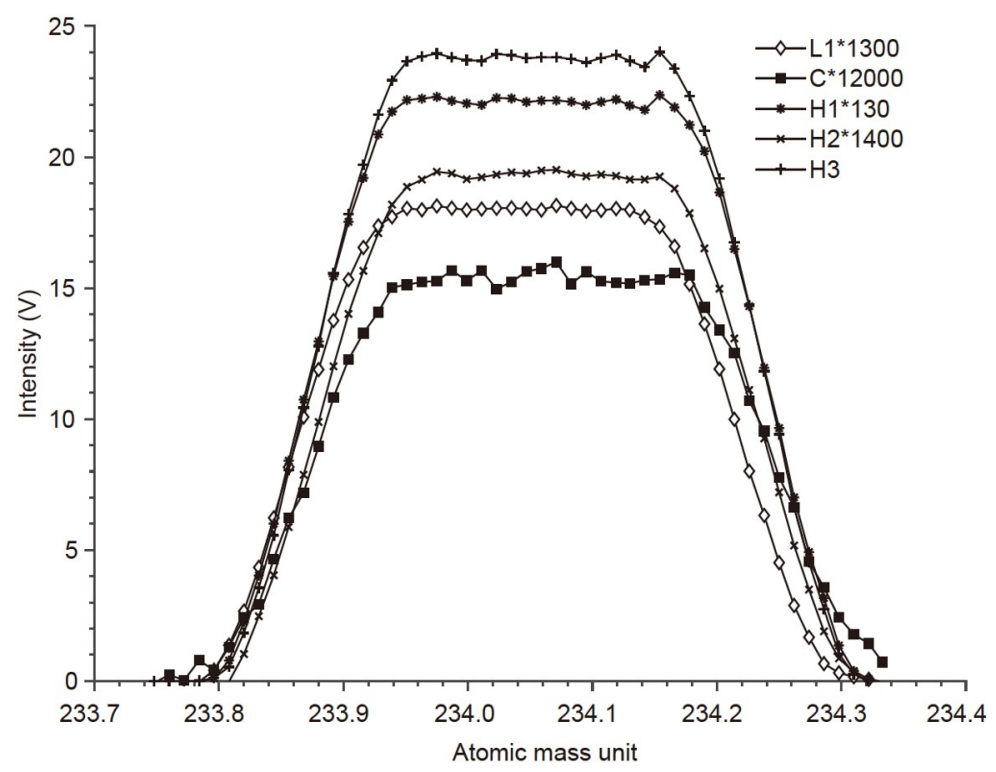

图 1 铀同位素的峰形

Figure 1 Peak shape of the analyzed U isotopes

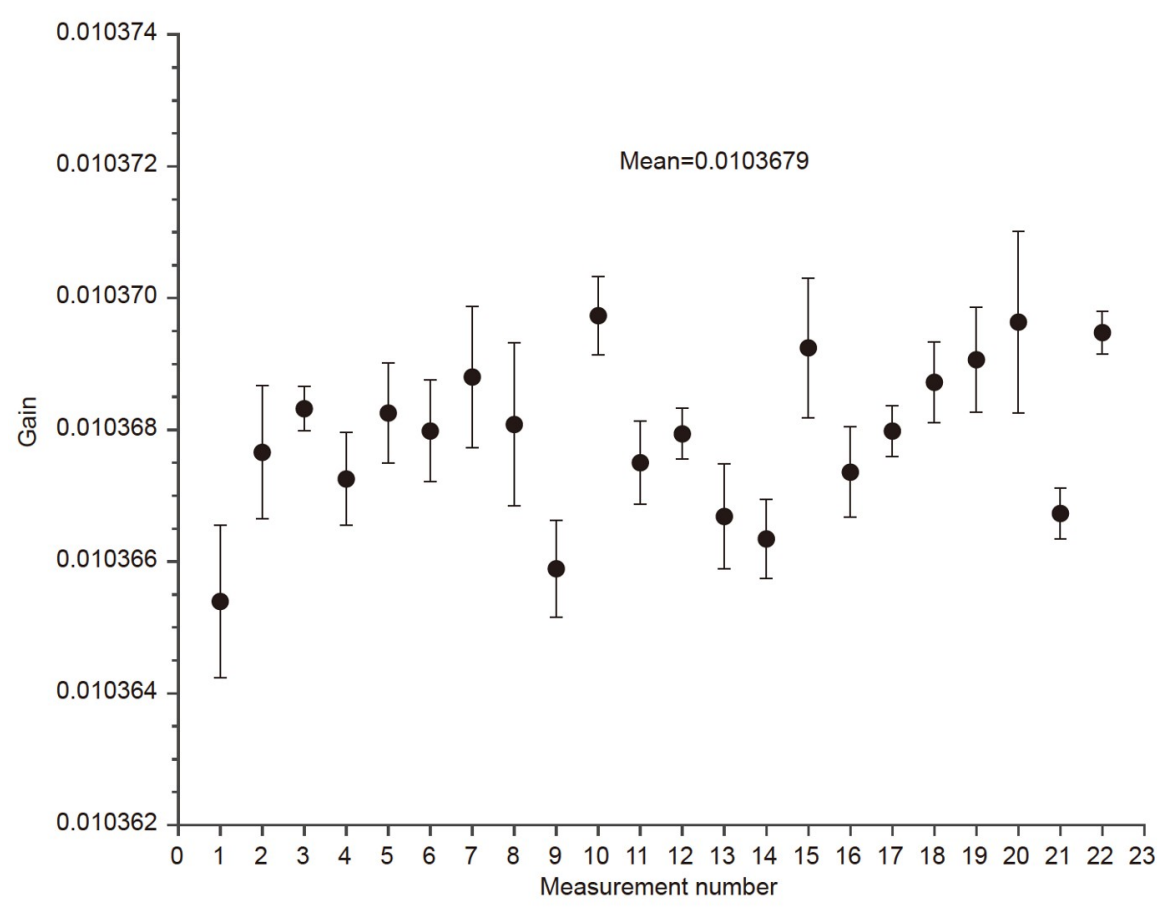

图 2 长期观测的 $10^{13} \Omega$ 放大器的法拉第杯增益(2021年6 10月, $\left.n=22\right)$

Figure 2 Long-term observed gain of a Faraday cup connected with a $10^{13} \Omega$ amplifier during the June and October of $2021(n=22)$

-0.979 和 $-0.830 \mathrm{mV}$. 如图3所示, $\mathrm{C}$ 位置的 $10^{13} \Omega$ 的法拉 第杯基线波动最大 $(\sim 41 \mu \mathrm{V}, 1 \mathrm{std})$, 其次为 $\mathrm{L} 1$ 和 $\mathrm{H} 2$ 位 置的两个 $10^{12} \Omega$ 的法拉第杯 $(\sim 11$ 和 $12 \mu \mathrm{V}), \mathrm{H} 1$ 位置的 $10^{11} \Omega$ 的法拉第杯基线最为稳定 $(\sim 3.7 \mu \mathrm{V})$. 然而, $\mathrm{H} 3$ 位
置的 $10^{11} \Omega$ 的法拉第杯基线稳定相对较差 $(\sim 10 \mu \mathrm{V})$, 这 可能是由于该位置长期用于测量高丰度的 ${ }^{238} \mathrm{U}$ 离子束.

在检测法拉第杯的基线之后再用SEM测量仪器的 本底. 长期的观测发现, 通过 $1 \mathrm{~mol} / \mathrm{L} \mathrm{HNO}_{3}+0.01 \mathrm{~mol} / \mathrm{L}$ 


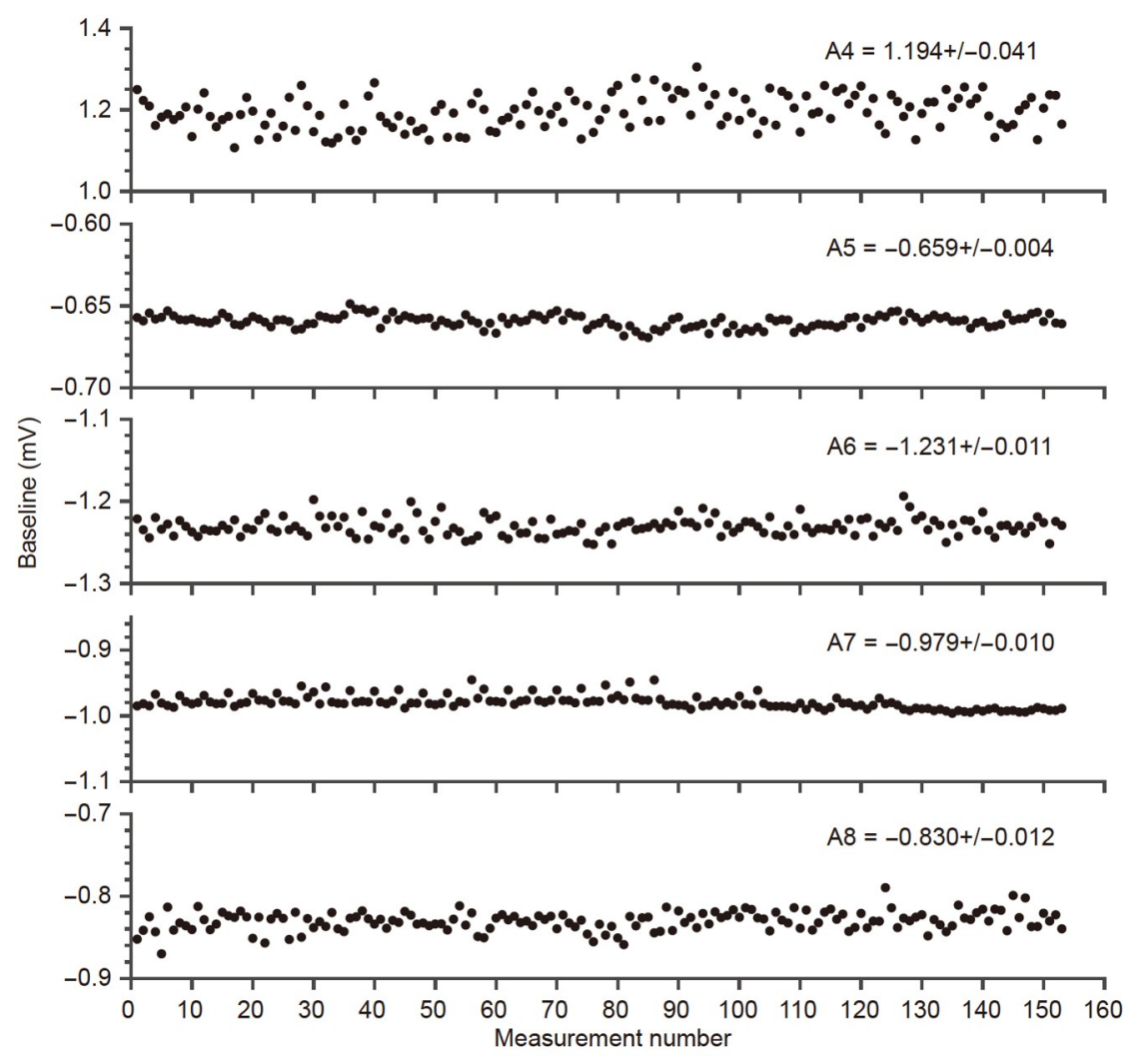

图 3 2021年8 9月法拉第杯的基线

Figure 3 Baseline of the Faraday cups observed during the August and September of 2021

$\mathrm{HF}$ 混合酸清洗 $3 \mathrm{~min},{ }^{238} \mathrm{U}$ 本底可降低到 $2 \sim 5 \mathrm{mV}$; 再经 过0.5 mol/ $\mathrm{L} \mathrm{HNO}_{3}+0.01 \mathrm{~mol} / \mathrm{L} \mathrm{HF}$ 混合酸清洗 $10 \mathrm{~min}$, 本底可降低到 $10^{-5}$ 的水平 $\left({ }^{238} \mathrm{U}<0.3 \mathrm{mV},{ }^{234} \mathrm{U}\right.$ 为 $\left.1 \sim 5 \mathrm{cps}\right)$.

\section{4 拖尾效应和氢化物校正}

离子在从离子源到检测器的传输过程中可能与仪 器内部残留气体分子或离子碰撞损失能量，并被磁场 以相对较小的半径偏转，从而在低质量一侧形成拖尾 信号. 为测量 ${ }^{238} \mathrm{U}$ 拖尾效应(tailing), 引人铀溶液 ${ }^{238} \mathrm{U}$ 信 号为 $30 \sim 40 \mathrm{~V})$, 用SEM扫描虚拟质量数为 237.05 、

$236.54 、 235.54 、 234.55 、 233.54$ 和 232.80 的离子束强 度(RPQ-off), 从而拟合出 ${ }^{238} \mathrm{U}$ 的拖尾效应. 铀的氢化物 (hydride) ${ }^{233} \mathrm{UH}^{+} 、{ }^{235} \mathrm{UH}^{+}$对 ${ }^{234} \mathrm{U}$ 和 ${ }^{236} \mathrm{U}$ 形成干扰信号. 为 校正这种干扰, 引人铀溶液 $\left({ }^{238} \mathrm{U}\right.$ 信号为 $\left.3 \sim 4 \mathrm{~V}\right)$, 用L1位 置的法拉第杯上测量 ${ }^{238} \mathrm{U}$, 同时在中心杯位置用SEM测 量虚拟质量数 239.05 的信号(RPQ-off). 将后者与前者 的信号强度之比视为铀氢化物干扰程度.

在测量过程中, 每隔5 10 h 测量一次仪器的拖尾效 应和氢化物干扰，将前后两次结果的平均值用于校正
其间测量的样品数据. 图4为 2021 年8 9月测量的 ${ }^{238} \mathrm{U}$ 在 ${ }^{236} \mathrm{U} 、{ }^{235} \mathrm{U} 、{ }^{234} \mathrm{U}$ 和 ${ }^{233} \mathrm{U}$ 峰上的拖尾程度，依次为 $\sim 1.08 、 \sim 0.47 、 \sim 0.25$ 和 $\sim 0.16 \mathrm{ppm}$. 长期观测显示铀的 氢化物干扰为 $2.14 \mathrm{ppm}$.

\section{5 质量歧视校正}

离子束在从离子源到探测器的传输过程中，较轻 的离子易受干扰而偏离飞行路径，使得较轻同位素与 较重同位素之比的测量值小于真实值，即质量歧视效 应. 采用指数法则 ${ }^{[32]}$ 校正铀同位素的质量歧视:

$R_{\mathrm{t}}=R_{\mathrm{m}}\left(\frac{M_{i}}{M_{j}}\right)^{\beta}$,

式中, $R_{\mathrm{m}} 、 R_{\mathrm{t}}$ 分别为同位素 $i / j$ 的测量值和真实值, $M_{i}$ 、 $M_{j}$ 分别为 $i$ 和 $j$ 的原子质量, $\beta$ 为质量歧视因子. 该因子由 校正拖尾和氢化物的 ${ }^{233} \mathrm{U}{ }^{236} \mathrm{U}$ 比值与已知值(1.01906) 相比较所得:

$\beta=\ln \left[\left(\frac{{ }^{233} \mathrm{U}}{{ }^{236} \mathrm{U}}\right)_{\mathrm{t}} /\left(\frac{{ }^{233} \mathrm{U}}{{ }^{236} \mathrm{U}}\right)_{\mathrm{m}}\right] / \ln \left(\frac{M_{233}}{M_{236}}\right)$. 


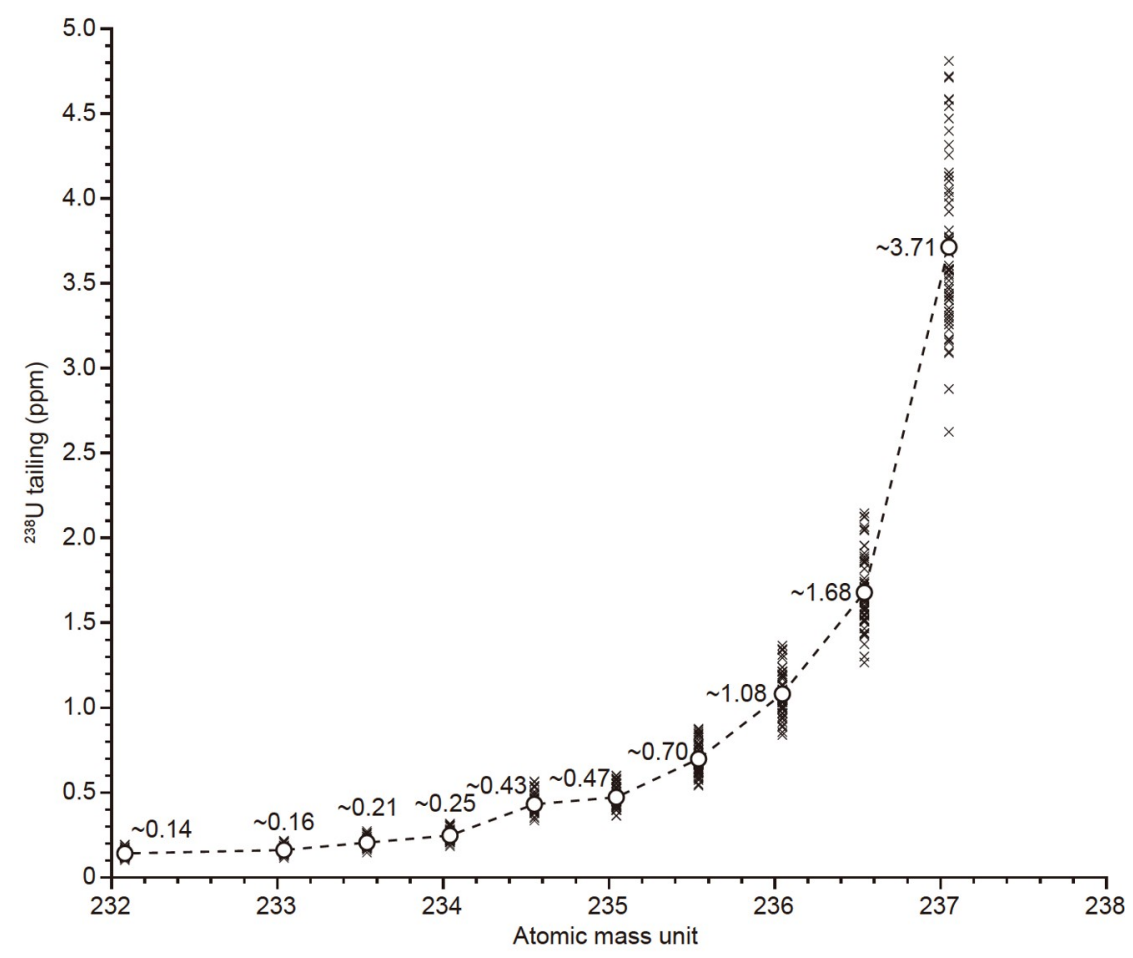

图 42021 年8 9月 ${ }^{238} \mathrm{U}$ 的拖尾效应

Figure $4{ }^{238} \mathrm{U}$ tailing effect measured during the August and September of 2021

\section{3 结果与讨论}

\section{1 标准样品HU-1}

HU-1(Harwell uraninite)是国际上广泛使用的铀系 衰变平衡的标准物质之一, 由前寒武纪氧化铀矿制备. 自20世纪90年代以来，国际上多家实验室采用TIMS和 MC-ICP-MS技术对HU-1的 ${ }^{234} \mathrm{U} /{ }^{238} \mathrm{U}$ 原子比进行高精度 分析, 测试结果非常一致, 为 $54.89 \times 10^{-6[5 \sim 7,20,21,24,33,34]}$. 近年来，多家实验室又对 $\mathrm{HU}-1$ 的 ${ }^{238} \mathrm{U} /{ }^{235} \mathrm{U}$ 原子比进行 了高精度测量. 2007年, Stirling等人 ${ }^{[21]}$ 首次用MC-ICP$\mathrm{MS}$ 分析 $\mathrm{HU}-1$ 的 ${ }^{238} \mathrm{U} /{ }^{235} \mathrm{U}$ 并得出原子比为 137.803 \pm 0.012 . 此后, Condon等人 ${ }^{[5]}$ 报道了 TIMS的测量结果为 $137.769 \pm 0.024$. Hiess等人 ${ }^{[6]}$ 同时报道了 TIMS和MCICP-MS的测试结果, 分别为 $137.760 \pm 0.022$ 和 137.755 \pm 0.022 . Cheng等人 ${ }^{[7]}$ 又报道了MC-ICP-MS的测试结果 为 $137.766 \pm 0.010$.

我们取少量HU-1溶液，加人IRMM-3636稀释剂与 之混匀 $\left({ }^{235} \mathrm{U} /{ }^{236} \mathrm{U}\right.$ 约为 1.5), 再用UTEVA树脂分离和纯 化铀. MC-ICP-MS的10次分析结果(表3)显示，HU-1 的 ${ }^{234} \mathrm{U} /{ }^{238} \mathrm{U}$ 原子比平均值为 $(54.90 \pm 0.03) \times 10^{-6}$ (或 $\delta^{234} \mathrm{U}=$ $-1.3 \pm 0.5)$, 与前人的测试结果一致. 我们测定的 ${ }^{238} U{ }^{235} U$
平均值为 $137.765 \pm 0.010$ (或 $\delta^{238} \mathrm{U}=-0.49 \pm 0.07$ ), 此值显 著低于Stirling等人 ${ }^{[21]}$ 的测试结果，而与其他已发表的 结果一致(图5).

\section{2 标准样品SRM950a}

SRM950a是国际氧化铀标准物质 $\left(\mathrm{U}_{3} \mathrm{O}_{8}, 99.94 \%\right.$ $\pm 0.02 \%)$ ，具有天然的铀同位素组成. Chen等人 ${ }^{[35]}$ 首次 用TIMS分析了SRM950a的铀同位素，发现该物质 的 ${ }^{234} \mathrm{U}{ }^{238} \mathrm{U}$ 为 $(53.66 \pm 0.16) \times 10^{-6}$, 比标准样品CRM-112a (又称SRM-960、NBL-112a或CRM-145)高了 16\% ，超 出 $2 \sigma$ 的分析误差范围. Condon等人 ${ }^{[5]}$ 用TIMS分析了 SRM950a的 ${ }^{238} \mathrm{U} /{ }^{235} \mathrm{U}$ 为 $137.847 \pm 0.024$. Goldmann等 人 $^{[36]}$ 又用MC-ICP-MS测定该比值为 $137.846 \pm 0.002$.

我们取少量的SRM950a溶液, 加人IRMM-3636稀 释剂与之混匀 ${ }^{235} \mathrm{U} /{ }^{236} \mathrm{U}$ 约为 1.1$)$, 之后用UTEVA树脂 分离和纯化铀. MC-ICP-MS的10次分析结果(表3)显示 该标准物质的 ${ }^{234} \mathrm{U} /{ }^{238} \mathrm{U}$ 原子比平均值为 $(53.88 \pm 0.03) \times$ $10^{-6}$ (或 $\left.\delta^{234} \mathrm{U}=-19.76 \pm 0.61\right) ;{ }^{238} \mathrm{U} /{ }^{235} \mathrm{U}$ 原子比的平均值 为 $137.844 \pm 0.005$ (或 $\delta^{238} \mathrm{U}=0.08 \pm 0.03$ ). 本文的测试结果 和已发表的数据都表明, SRM950a的 ${ }^{234} \mathrm{U} /{ }^{238} \mathrm{U}$ 比值显著 高于 CRM-112a $(52.852 \pm 0.015)^{[7]}$, 而 ${ }^{238} \mathrm{U} /{ }^{235} \mathrm{U}$ 比值与 
CRM-112a(137.832 \pm 0.015$)^{[7]}$ 在测试误差内一致(图5).

\section{3 标准样品GBW04412}

GBW04412是我国铀系年龄标准物质, 由采自贵州 犀牛洞具有清晰生长纹层的文石制备, 铀含量为 $\sim 9.3 \mathrm{ppm}$ ，年代为 $\sim 85 \mathrm{ka}$. 夏明等人 ${ }^{[40]}$ 采用 $\alpha$-能谱技术 测定了该标准物质的 ${ }^{234} \mathrm{U} /{ }^{238} \mathrm{U}$ 活度比为 $1.85 \pm 0.07$. Peng 等人 ${ }^{[37]}$ 和 $\mathrm{Ma}$ 等人 ${ }^{[38]}$ 分别用TIMS 分析了该标准物质, ${ }^{234} \mathrm{U} /{ }^{238} \mathrm{U}$ 原子比分别为 $(102.08 \pm 0.39) \times 10^{-6}$ 和 $(101.86$ $\pm 0.16) \times 10^{-6}$. 彭子成等人 ${ }^{[39]}$ 又用TIMS和MC-ICP-MS两 种技术分析了该标准物质, ${ }^{234} \mathrm{U} /{ }^{238} \mathrm{U}$ 测试结果分别为 $(101.84 \pm 0.16) \times 10^{-6}$ 和 $(101.86 \pm 0.05) \times 10^{-6}$. Wang和 $\mathrm{You}^{[25]}$ 又用MC-ICP-MS标准样品-样品交叉测量方法分 析了该标准物质, ${ }^{234} \mathrm{U} /{ }^{238} \mathrm{U} 、{ }^{238} \mathrm{U} /{ }^{235} \mathrm{U}$ 原子比分别为 $(101.41 \pm 0.329) \times 10^{-6}$ 和 $137.720 \pm 0.190$.

我们称取 8 个 GBW04412粉末样品, 每个样品为 $11 \sim 14 \mathrm{mg}$, 分别用 $7 \mathrm{~mol} / \mathrm{L} \mathrm{HNO}_{3}$ 溶解并加人IRMM3636稀释剂. 将稀释剂与样品溶液加热混匀之后 $\left({ }^{235} \mathrm{U} /\right.$ ${ }^{236} \mathrm{U}$ 约为 4.3 ), 再用UTEVA树脂分离和纯化铀. MC-
ICP-MS测试结果(表3)显示, ${ }^{234} U /{ }^{238} U 、{ }^{238} U /{ }^{235} U$ 原子 比的平均值分别为 $(101.66 \pm 0.04) \times 10^{-6}$ (或 $\delta^{234} \mathrm{U}=849.4$ \pm 0.7 )和 $137.786 \pm 0.018$ (或 $\delta^{238} \mathrm{U}=-0.33 \pm 0.13$ ). 新的测试 结果与上述已发表的结果在误差范围内一致且精度显 著提高(图5).

\section{4 标准样品GBW04428}

GBW04428是由核工业北京地质研究院研制的铀 同位素活度比溶液标准物质。通过国内多家单位的 $\alpha$ 能谱、ICP-MS和TIMS分析测试, GBW04428浓度被标 定为 $0.999 \mathrm{mg} \mathrm{U} / \mathrm{mL},{ }^{234} \mathrm{U} /{ }^{238} \mathrm{U}$ 活度比为 $0.99 \pm 0.01$. 然 而，目前在文献中还没有对该标准物质铀同位素组成 的精确研究. 我们取稀释的GBW04428, 加人IRMM3636稀释剂与之混匀 ${ }^{235} \mathrm{U}^{236} \mathrm{U}$ 约为 1.4$)$, 之后进行 $\mathrm{MC}$ ICP-MS铀同位素分析. 10次测试结果(表3)显示，该标 准物质的 ${ }^{234} \mathrm{U} /{ }^{238} \mathrm{U}$ 原子比平均值为 $(53.86 \pm 0.03) \times 10^{-6}$ (或 $\delta^{234} \mathrm{U}=-20.2 \pm 0.5$ ), 接近标定值的最低限. 同时, 测试 结果显示 $\mathrm{GBW} 04428$ 的 ${ }^{238} \mathrm{U} /{ }^{235} \mathrm{U}$ 为 $137.995 \pm 0.008$ (或 $\delta^{238} \mathrm{U}=1.18 \pm 0.06$ ), 显著高于本文的其他标准物质(图5).
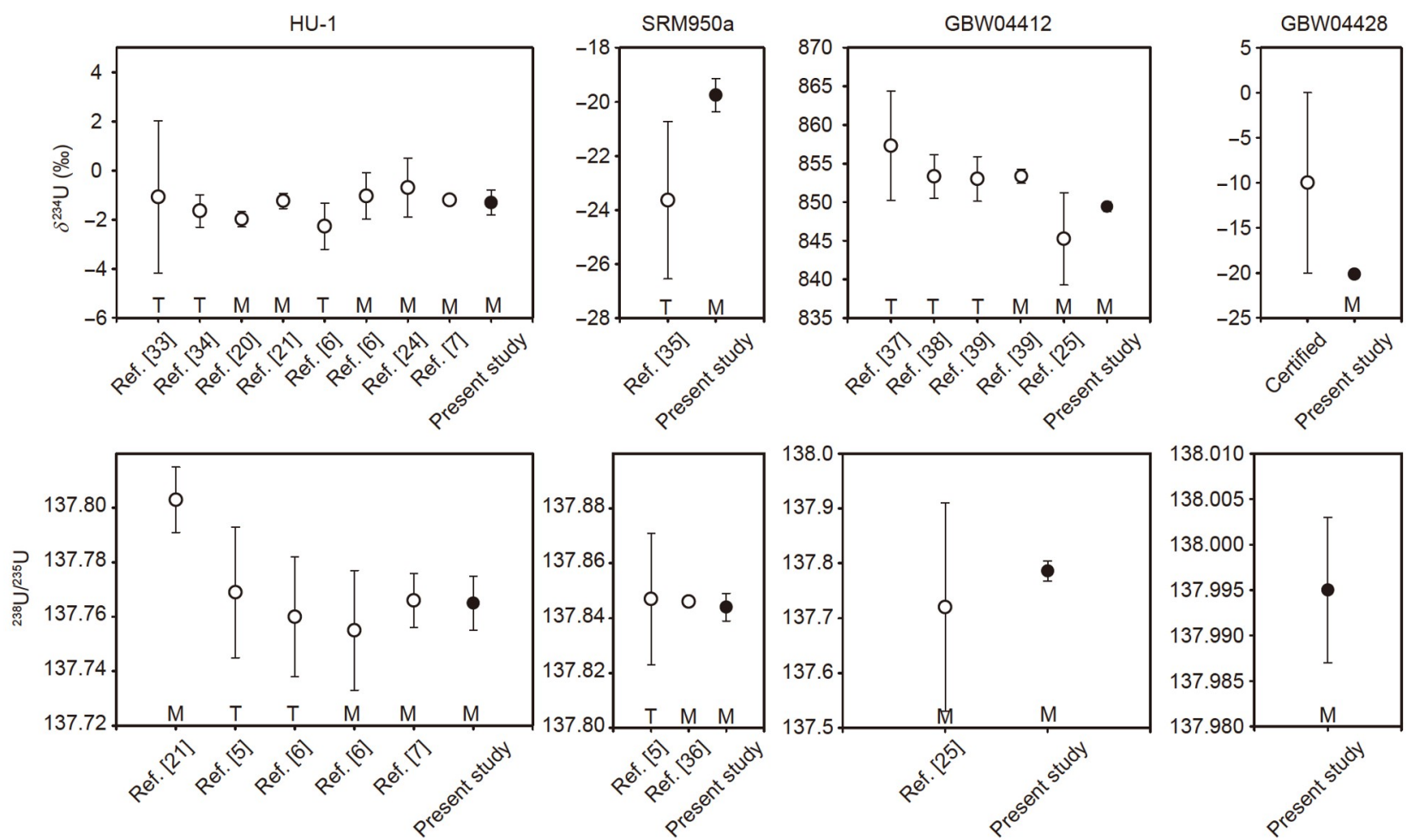

图 5 国际与国内铀标准物质的 ${ }^{234} \mathrm{U} /{ }^{238} \mathrm{U}$ 和 ${ }^{238} \mathrm{U} /{ }^{235} \mathrm{U}( \pm 2 \sigma)$ 对比. T, TIMS; M, MC-ICP-MS. $\left.\delta^{234} \mathrm{U}=\left[\left({ }^{234} \mathrm{U} /{ }^{238} \mathrm{U}\right)_{\text {smp }} /\left(^{234} \mathrm{U} /{ }^{238} \mathrm{U}\right)_{\mathrm{se.}}-1\right] \times 1000,{ }^{234} \mathrm{U} /{ }^{238} \mathrm{U}\right)_{\mathrm{se.}}=$ $54.970 \times 10^{-6[7]}$

Figure 5 Comparison of measurements of ${ }^{234} \mathrm{U} /{ }^{238} \mathrm{U}$ and ${ }^{238} \mathrm{U} /{ }^{235} \mathrm{U}( \pm 2 \sigma)$ in the international and national standards. T, TIMS, M, MC-ICP-MS. $\delta^{234} \mathrm{U}=$ $\left.\left[\left({ }^{234} \mathrm{U} /^{238} \mathrm{U}\right)_{\text {smp }} /\left(^{234} \mathrm{U} /{ }^{238} \mathrm{U}\right)_{\text {s.e. }}-1\right] \times 1000,{ }^{234} \mathrm{U} /{ }^{238} \mathrm{U}\right)_{\text {s.e. }}=54.970 \times 10^{-6677]}$ 
表 3 国际和国内铀标准物质的 ${ }^{234} U /{ }^{238} U 、{ }^{238} U /{ }^{235} U$ 值

Table 3 Measurements of ${ }^{234} \mathrm{U} /{ }^{238} \mathrm{U}$ and ${ }^{238} \mathrm{U} /{ }^{235} \mathrm{U}$ in the international and national standards

\begin{tabular}{|c|c|c|c|c|}
\hline 标准样品 & ${ }^{234} \mathrm{U} /{ }^{238} \mathrm{U}\left(\times 10^{-6}\right)$ & $\delta^{234} \mathrm{U}(\% \mathrm{o})^{\mathrm{a})}$ & ${ }^{238} U /{ }^{235} U$ & $\left.\delta^{238} \mathrm{U}(\%)\right)^{\mathrm{b})}$ \\
\hline \multirow[t]{10}{*}{ HU-1 } & $54.914 \pm 0.026$ & $-1.013 \pm 0.465$ & $137.758 \pm 0.006$ & $-0.537 \pm 0.041$ \\
\hline & $54.901 \pm 0.027$ & $-1.249 \pm 0.485$ & $137.755 \pm 0.006$ & $-0.559 \pm 0.040$ \\
\hline & $54.877 \pm 0.028$ & $-1.699 \pm 0.505$ & $137.766 \pm 0.006$ & $-0.478 \pm 0.042$ \\
\hline & $54.884 \pm 0.026$ & $-1.559 \pm 0.481$ & $137.770 \pm 0.008$ & $-0.448 \pm 0.059$ \\
\hline & $54.889 \pm 0.027$ & $-1.470 \pm 0.492$ & $137.762 \pm 0.007$ & $-0.508 \pm 0.054$ \\
\hline & $54.892 \pm 0.025$ & $-1.421 \pm 0.459$ & $137.768 \pm 0.005$ & $-0.464 \pm 0.039$ \\
\hline & $54.898 \pm 0.026$ & $-1.304 \pm 0.464$ & $137.768 \pm 0.006$ & $-0.466 \pm 0.043$ \\
\hline & $54.912 \pm 0.025$ & $-1.057 \pm 0.460$ & $137.767 \pm 0.005$ & $-0.473 \pm 0.039$ \\
\hline & $54.920 \pm 0.022$ & $-0.906 \pm 0.397$ & $137.766 \pm 0.007$ & $-0.476 \pm 0.048$ \\
\hline & $54.894 \pm 0.018$ & $-1.386 \pm 0.335$ & $137.770 \pm 0.006$ & $-0.453 \pm 0.045$ \\
\hline 均值 & $54.898 \pm 0.028$ & $-1.306 \pm 0.506$ & $137.765 \pm 0.010$ & $-0.486 \pm 0.073$ \\
\hline \multirow[t]{10}{*}{ SRM950a } & $53.900 \pm 0.027$ & $-19.459 \pm 0.489$ & $137.843 \pm 0.005$ & $0.081 \pm 0.033$ \\
\hline & $53.898 \pm 0.023$ & $-19.494 \pm 0.410$ & $137.841 \pm 0.005$ & $0.063 \pm 0.036$ \\
\hline & $53.877 \pm 0.022$ & $-19.881 \pm 0.407$ & $137.841 \pm 0.005$ & $0.068 \pm 0.038$ \\
\hline & $53.870 \pm 0.023$ & $-20.011 \pm 0.410$ & $137.846 \pm 0.004$ & $0.104 \pm 0.029$ \\
\hline & $53.890 \pm 0.022$ & $-19.649 \pm 0.408$ & $137.841 \pm 0.005$ & $0.067 \pm 0.035$ \\
\hline & $53.875 \pm 0.021$ & $-19.915 \pm 0.380$ & $137.843 \pm 0.006$ & $0.076 \pm 0.040$ \\
\hline & $53.916 \pm 0.029$ & $-19.174 \pm 0.525$ & $137.847 \pm 0.006$ & $0.112 \pm 0.040$ \\
\hline & $53.862 \pm 0.024$ & $-20.152 \pm 0.433$ & $137.845 \pm 0.005$ & $0.093 \pm 0.034$ \\
\hline & $53.878 \pm 0.021$ & $-19.859 \pm 0.389$ & $137.844 \pm 0.006$ & $0.084 \pm 0.040$ \\
\hline & $53.872 \pm 0.020$ & $-19.970 \pm 0.356$ & $137.845 \pm 0.005$ & $0.094 \pm 0.037$ \\
\hline 均值 & $53.884 \pm 0.033$ & $-19.756 \pm 0.606$ & $137.844 \pm 0.005$ & $0.084 \pm 0.033$ \\
\hline \multirow[t]{8}{*}{ GBW04412 } & $101.670 \pm 0.020$ & $849.529 \pm 0.356$ & $137.791 \pm 0.010$ & $-0.298 \pm 0.069$ \\
\hline & $101.650 \pm 0.025$ & $849.220 \pm 0.459$ & $137.795 \pm 0.009$ & $-0.266 \pm 0.068$ \\
\hline & $101.630 \pm 0.026$ & $848.890 \pm 0.475$ & $137.789 \pm 0.008$ & $-0.315 \pm 0.060$ \\
\hline & $101.650 \pm 0.024$ & $849.224 \pm 0.432$ & $137.770 \pm 0.009$ & $-0.452 \pm 0.062$ \\
\hline & $101.650 \pm 0.024$ & $849.208 \pm 0.429$ & $137.781 \pm 0.009$ & $-0.372 \pm 0.063$ \\
\hline & $101.670 \pm 0.029$ & $849.547 \pm 0.537$ & $137.797 \pm 0.009$ & $-0.257 \pm 0.062$ \\
\hline & $101.690 \pm 0.024$ & $849.846 \pm 0.437$ & $137.791 \pm 0.007$ & $-0.297 \pm 0.049$ \\
\hline & $101.690 \pm 0.025$ & $849.865 \pm 0.462$ & $137.779 \pm 0.009$ & $-0.385 \pm 0.065$ \\
\hline 均值 & $101.663 \pm 0.042$ & $849.416 \pm 0.681$ & $137.786 \pm 0.018$ & $-0.330 \pm 0.134$ \\
\hline \multirow[t]{10}{*}{ GBW04428 } & $53.848 \pm 0.027$ & $-20.418 \pm 0.486$ & $138.004 \pm 0.005$ & $1.244 \pm 0.039$ \\
\hline & $53.843 \pm 0.022$ & $-20.504 \pm 0.396$ & $137.996 \pm 0.004$ & $1.188 \pm 0.032$ \\
\hline & $53.858 \pm 0.020$ & $-20.224 \pm 0.372$ & $137.999 \pm 0.006$ & $1.210 \pm 0.040$ \\
\hline & $53.870 \pm 0.021$ & $-20.011 \pm 0.384$ & $137.995 \pm 0.006$ & $1.181 \pm 0.045$ \\
\hline & $53.873 \pm 0.021$ & $-19.955 \pm 0.389$ & $137.992 \pm 0.006$ & $1.158 \pm 0.042$ \\
\hline & $53.860 \pm 0.024$ & $-20.191 \pm 0.429$ & $137.994 \pm 0.006$ & $1.172 \pm 0.044$ \\
\hline & $53.880 \pm 0.024$ & $-19.836 \pm 0.437$ & $137.993 \pm 0.006$ & $1.167 \pm 0.047$ \\
\hline & $53.879 \pm 0.025$ & $-19.851 \pm 0.461$ & $137.997 \pm 0.005$ & $1.195 \pm 0.034$ \\
\hline & $53.848 \pm 0.023$ & $-20.411 \pm 0.421$ & $137.991 \pm 0.006$ & $1.155 \pm 0.043$ \\
\hline & $53.865 \pm 0.028$ & $-20.105 \pm 0.514$ & $137.991 \pm 0.006$ & $1.152 \pm 0.044$ \\
\hline 均值 & $53.862 \pm 0.026$ & $-20.151 \pm 0.480$ & $137.995 \pm 0.008$ & $1.182 \pm 0.057$ \\
\hline
\end{tabular}

a) $\delta^{234} U=\left[\left({ }^{234} U /{ }^{238} U\right)_{\text {smp }} /\left({ }^{234} U /{ }^{238} U\right)_{\text {s.e. }}-1\right] \times 1000$, 其中 $\left({ }^{234} U{ }^{238} U\right)_{\text {s.e. }}$ 为放射性衰变久期平衡状态下 ${ }^{234} U$ 与 ${ }^{238} U$ 的原子比值, $54.970 \times 10^{-6[7] ; ~ b) ~}$ $\delta^{238} \mathrm{U}=\left[\left({ }^{238} \mathrm{U} /{ }^{235} \mathrm{U}\right)_{\mathrm{smp}} /\left({ }^{238} \mathrm{U} /{ }^{235} \mathrm{U}\right)_{\mathrm{CRM}-112 \mathrm{a}}-1\right] \times 1000$, 其中 $\left({ }^{238} \mathrm{U} /{ }^{235} \mathrm{U}\right)_{\mathrm{CRM}-112 \mathrm{a}}$ 为国际铀标准样品CRM-112a的 ${ }^{238} \mathrm{U} /{ }^{235} \mathrm{U}=137.832^{[6,7]}$ 


\section{4 结论}

我们利用MC-ICP-MS灵活的检测系统，组合使用 $10^{11} 、 10^{12}$ 和 $10^{13} \Omega$ 高阻信号放大器, 建立了铀同位素 静态分析的新方法. 在通常情况之下, 仪器的传输效率 可达到 $2 \%$, 使用 $20 \sim 30 \mathrm{ng}$ 铀便可以同时精确测量 ${ }^{234} \mathrm{U} /$ ${ }^{238} \mathrm{U}$ 和 ${ }^{238} \mathrm{U} /{ }^{235} \mathrm{U}$. 对国际标准样品HU-1和SRM950a的测 量表明, 这两种物质中的 ${ }^{234} \mathrm{U} /{ }^{238} \mathrm{U}$ 原子比分别为(54.90土 $0.03) \times 10^{-6}$ 和 $(53.88 \pm 0.03) \times 10^{-6} ;{ }^{238} \mathrm{U} /{ }^{235} \mathrm{U}$ 分别为 $137.765 \pm 0.010$ 和 $137.844 \pm 0.005$. 我们对国内标准样品
GBW04412和GBW04428的测量表明，这两种物质中 的 ${ }^{234} \mathrm{U} /{ }^{238} \mathrm{U}$ 原子比分别为 $(101.66 \pm 0.04) \times 10^{-6}$ 和(53.86 $0.03) \times 10^{-6} ;{ }^{238} \mathrm{U} /{ }^{235} \mathrm{U}$ 分别为 $137.786 \pm 0.018$ 和 $137.995 \pm$ 0.008. 其中, 标准样品GBW04428的铀同位素是首次用 MC-ICP-MS高精度测量. 本文的分析测试结果与国内 外已发表的数据高度一致, 证实了我们新方法的可靠 性. 此外, 新方法可以根据样品的铀含量和对分析精度 的要求灵活调整高阻放大器的排列方式, 比如用 $10^{10} \Omega$ 的法拉第杯接收 ${ }^{238} \mathrm{U}$, 可把信号强度的测试上限提高 10 倍, 从而可以进一步提高铀同位素的测试精度.

致谢感谢审稿人提供重要的修改意见, 感谢南京师范大学地理科学学院石笋同位素实验室的所有老师和研究生为Neptune MC-ICP-MS的维护和运行所作的努力.

\section{参考文献}

1 Meija J, Coplen T B, Berglund M, et al. Isotopic compositions of the elements 2013 (IUPAC Technical Report). Pure Appl Chem, 2016, 88: 293306

2 Jaffey A H, Flynn K F, Glendenin L E, et al. Precision measurement of half-lives and specific activities of ${ }^{235}$ U and ${ }^{238} \mathrm{U}$. Phys Rev C, 1971, 4: 1889-1906

3 Steiger R H, Jäger E. Subcommission on geochronology: Convention on the use of decay constants in geo- and cosmochronology. Earth Planet Sci Lett, 1977, 36: 359-362

4 Goldstein S J. Techniques for measuring uranium-series nuclides: 1992-2002. Rev Mineral Geochem, 2003, 52: 23-57

5 Condon D J, McLean $\mathrm{N}$, Noble $\mathrm{S}$ R, et al. Isotopic composition $\left({ }^{238} \mathrm{U} /{ }^{235} \mathrm{U}\right)$ of some commonly used uranium reference materials. Geochim Cosmochim Acta, 2010, 74: 7127-7143

6 Hiess J, Condon D J, McLean N, et al. ${ }^{238} \mathrm{U} /{ }^{235} \mathrm{U}$ systematics in terrestrial uranium-bearing minerals. Science, 2012, 335: 1610-1614

7 Cheng H, Edwards R L, Shen C C, et al. Improvements in ${ }^{230} \mathrm{Th}$ dating, ${ }^{230} \mathrm{Th}$ and ${ }^{234} \mathrm{U}$ half-life values, and U-Th isotopic measurements by multicollector inductively coupled plasma mass spectrometry. Earth Planet Sci Lett, 2013, 371-372: 82-91

8 Dunk R M, Mills R A, Jenkins W J. A reevaluation of the oceanic uranium budget for the Holocene. Chem Geol, 2002, 190: 45-67

9 Robinson L F, Belshaw N S, Henderson G M. U and Th concentrations and isotope ratios in modern carbonates and waters from the Bahamas. Geochim Cosmochim Acta, 2004, 68: 1777-1789

10 Osmond J K, Cowart J B. Ground water. In: Ivanovich M, Harmon R S, eds. Uranium Series Disequilibrium: Application to Environmental Problems. Oxford: Oxford Science Publications, 1992. 204-245

11 Andersen M B, Stirling C H, Porcelli D, et al. The tracing of riverine U in Arctic seawater with very precise ${ }^{234} \mathrm{U} /{ }^{238} \mathrm{U}$ measurements. Earth Planet Sci Lett, 2007, 259: 171-185

12 Andersen M B. Precise determination of the open ocean ${ }^{234} \mathrm{U}^{238} \mathrm{U}$ composition. Geochem Geophys Geosyst, 2010, 11: Q12003

13 Edwards R L, Chen J H, Wasserburg G J. ${ }^{238} \mathrm{U}_{-}{ }^{234} \mathrm{U}_{-}{ }^{230} \mathrm{Th}_{-}{ }^{232}$ Th systematics and the precise measurement of time over the past 500000 years. Earth Planet Sci Lett, 1987, 81: 175-192

14 Woodhead J, Reisz R, Fox D, et al. Speleothem climate records from deep time? Exploring the potential with an example from the Permian. Geology, 2010, 38: 455-458

15 DePaolo D J, Maher K, Christensen J N, et al. Sediment transport time measured with U-series isotopes: Results from ODP North Atlantic drift site 984. Earth Planet Sci Lett, 2006, 248: 394-410

16 Li L, Liu X, Li T, et al. Uranium comminution age tested by the eolian deposits on the Chinese Loess Plateau. Earth Planet Sci Lett, 2017, 467: 6471

17 Ivanovich M, Harmon R S. Uranium-series Disequilibrium: Applications to Earth, Marine, and Environmental Sciences. Oxford: Clarendon Press, 1992

18 Bourdon B. Introduction to U-series geochemistry. Rev Mineral Geochem, 2003, 52: 1-21 
19 Luo X, Rehkämper M, Lee D C, et al. High precision ${ }^{230} \mathrm{Th} /{ }^{232} \mathrm{Th}$ and ${ }^{234} \mathrm{U} /{ }^{238} \mathrm{U}$ measurements using energyfiltered ICP magnetic sector multiple collector mass spectrometry. Int J Mass Spectrom Ion Process, 1997, 171: 105-117

20 Andersen M B, Stirling C H, Potter E K, et al. Toward epsilon levels of measurement precision on ${ }^{234} \mathrm{U} /{ }^{238} \mathrm{U}$ by using MC-ICPMS. Int J Mass Spectrom, 2004, 237: 107-118

21 Stirling C H, Andersen M B, Potter E K, et al. Low-temperature isotopic fractionation of uranium. Earth Planet Sci Lett, 2007, 264: 208-225

22 Weyer S, Anbar A D, Gerdes A, et al. Natural fractionation of ${ }^{238} \mathrm{U} /{ }^{235} \mathrm{U}$. Geochim Cosmochim Acta, 2008, 72: 345-359

23 Brennecka G A, Borg L E, Hutcheon I D, et al. Natural variations in uranium isotope ratios of uranium ore concentrates: Understanding the ${ }^{238} \mathrm{U} /{ }^{235} \mathrm{U}$ fractionation mechanism. Earth Planet Sci Lett, 2010, 291: 228-233

24 Shen C C, Wu C C, Cheng H, et al. High-precision and high-resolution carbonate ${ }^{230}$ Th dating by MC-ICP-MS with SEM protocols. Geochim Cosmochim Acta, 2012, 99: 71-86

25 Wang R M, You C F. Precise determination of U isotopic compositions in low concentration carbonate samples by MC-ICP-MS. Talanta, 2013, 107: $67-73$

26 Liao Z B, Shao Q F, Li C H, et al. Measurement of U/Th isotopic compositions in stalagmites for ${ }^{230} \mathrm{Th} / \mathrm{U}$ geochronology using MC-ICP-MS by standard sample bracketing method (in Chinese). J Chin Mass Spectrom Soc, 2018, 39: 48-62 [廖泽波, 邵庆丰, 李春华, 等. MC-ICP-M标样-样品 交叉测试法测定石笋样品的 ${ }^{230} \mathrm{Th} / \mathrm{U}$ 年龄. 质谱学报, 2018, 39: 48-62]

27 Shao Q F, Li C H, Huang M J, et al. Interactive programs of MC-ICPMS data processing for ${ }^{230} \mathrm{Th} / \mathrm{U}$ geochronology. Quat Geochronol, 2019, 51: $43-52$

28 Li C H, Huang M J, Liao Z B, et al. U-Th isotopic measurements by MC-ICP-MS with multi-static method (in Chinese). J Chin Mass Spectrom Soc, 2019, 40: 209-221 [李春华, 黄孟杰, 廖泽波, 等. MC-ICP-MS两步静态法测量U-Th同位素. 质谱学报, 2019, 40: 209-221]

29 Koornneef J M, Bouman C, Schwieters J B, et al. Measurement of small ion beams by thermal ionisation mass spectrometry using new $10^{13}$ Ohm resistors. Anal Chim Acta, 2014, 819: 49-55

30 Breton T, Lloyd N S, Trinquier A, et al. Improving precision and signal/noise ratios for MC-ICP-MS. Procedia Earth Planet Sci, 2015, 13: 240-243

31 Richter S, Alonso-Munoz A, Eykens R, et al. The isotopic composition of natural uranium samples-Measurements using the new $n\left({ }^{233} \mathrm{U}\right) / n\left({ }^{236} \mathrm{U}\right)$ double spike IRMM-3636. Int J Mass Spectrom, 2008, 269: 145-148

32 Russell W A, Papanastassiou D A, Tombrello T A. Ca isotope fractionation on the earth and other solar system materials. Geochim Cosmochim Acta, 1978, 42: 1075-1090

33 Ludwig K R, Simmons K R, Szabo B J, et al. Mass-spectrometric ${ }^{230} \mathrm{Th}_{-}{ }^{234} \mathrm{U}_{-}{ }^{238} \mathrm{U}$ dating of the devils hole calcite vein. Science, 1992, 258: 284287

34 Cheng H, Edwards R L, Hoff J, et al. The half-lives of uranium-234 and thorium-230. Chem Geol, 2000, 169: 17-33

35 Chen J H, Lawrence R E, Wasserburg G J. ${ }^{238} \mathrm{U},{ }^{234} \mathrm{U}$ and ${ }^{232} \mathrm{Th}$ in seawater. Earth Planet Sci Lett, 1986, 80: 241-251

36 Goldmann A, Brennecka G, Noordmann J, et al. The uranium isotopic composition of the Earth and the Solar System. Geochim Cosmochim Acta, 2015, 148: 145-158

37 Peng Z C, Wang Z R, Sun W D, et al. High-precision timing of the quaternary standard samples with thermal ionization mass spectrometry (TIMS) U-series method. Chin Sci Bull, 1998, 44: 937-941

38 Ma Z B, Xia M, Zhang C, et al. High-precision U-series dating of Holocene corals from South China sea by thermal ionization mass spectrometry (TIMS). Chin Sci Bull, 1999, 44: 937-941

39 Peng Z C, He J F, Luo X Z, et al. High-precision timing of Nanhai coral by using MC-ICP-MS and TIMS techniques and its paleo-environmental significance (in Chinese). Nucl Sci Tech, 2004, 27: 469-474 [彭子成, 贺剑峰, 罗晓忠, 等. 南海珊瑚高精度热电离质谱和多接收电感耦合等离 子体质谱铀系年龄和古环境意义. 核技术, 2004, 27: 469-474]

40 Xia M, Zhang C H, Ma Z B. Results and discussion of uranium series domestic comparison program (in Chinese). Sci China Ser B, 1986, 16: 417427 [夏明, 张承蕙, 马志邦. 铀系国内对比计划的结果和讨论. 中国科学B辑, 1986, 16: 417-427] 


\title{
High-precision MC-ICP-MS static measurements of uranium isotopes using Faraday cups
}

\author{
Mangu Jin ${ }^{1,2}$, Yanuo Jiao ${ }^{1,2}$, Yue Liu ${ }^{1,2}$, Qingfeng Shao ${ }^{1,2^{*}}$, Xinggong Kong ${ }^{1,2}$ \& Yongjin Wang ${ }^{1,2}$ \\ ${ }^{1}$ Key Laboratory of Virtual Geographic Environment, Ministry of Education, School of Geography, Nanjing Normal University, Nanjing 210023, China; \\ 2 Jiangsu Center for Collaborative Innovation in Geographical Information Resource Development and Application, Nanjing 210023, China \\ * Corresponding author, E-mail: qingfengshao@njnu.edu.cn
}

High-precision measurements of ${ }^{238} \mathrm{U} /{ }^{235} \mathrm{U}$ and ${ }^{234} \mathrm{U} /{ }^{238} \mathrm{U}$ atomic ratios are essential for $\mathrm{U}-\mathrm{Pb}$ and ${ }^{230} \mathrm{Th} / \mathrm{U}$ dating, as well as for a wide range of Earth and environmental studies. The ${ }^{238} \mathrm{U} /{ }^{235} \mathrm{U}$ ratio of a natural material has been considered to be a constant of 137.88 since the 1970s. However, recent advances in analytical techniques, such as TIMS (Thermal Ionization Mass Spectrometry) and MC-ICP-MS (Multiple Collector Inductively Coupled Plasma Mass Spectrometry), allowed the measurement of ${ }^{238} \mathrm{U} /{ }^{235} \mathrm{U}$ ratios at a precision of $10^{-5}$, revealing up to $5 \%$ variations in terrestrial ${ }^{238} \mathrm{U} /{ }^{235} \mathrm{U}$ ratios. The ${ }^{234} \mathrm{U} /{ }^{238} \mathrm{U}$ ratios are commonly observed at the $10^{-4}-10^{-5}$ levels in the natural environment. These large differences in abundance make it impossible to simultaneously measure U isotopes in Faraday cups connected to the standard $10^{11} \Omega$ current amplifiers using the MC-ICP-MS. To obtain epsilon precision levels in the ${ }^{234} \mathrm{U} /{ }^{238} \mathrm{U}$ measurements, previous studies employed a $10^{9}$ or $10^{10} \Omega$ amplifier for the ${ }^{238} \mathrm{U}$ Faraday cup and a $10^{11} \Omega$ amplifier for the ${ }^{234} \mathrm{U}$ Faraday cup. However, these methods require a large quantity of sample material of $400-1000 \mathrm{ng}{ }^{238} \mathrm{U}$, as the $10^{11} \Omega$ amplifier has a relatively low signal/noise ratio. In 2014, Thermo Fisher Scientific launched a new Faraday cup current amplifier with a $10^{13} \Omega$ feedback resistor, which offers a signal/noise ratio improved by a factor of five compared with standard $10^{11} \Omega$ amplifiers. Therefore, we are attempting to use the new $10^{13} \Omega$ amplifier in combination with the $10^{11}$ and $10^{12} \Omega$ amplifiers to perform high-precision static measurements of $U$ isotopes with Faraday cups using a small amount of sample. In the present study, we simultaneously used a $10^{13} \Omega$ amplifier, two $10^{12} \Omega$ amplifiers, and two $10^{11} \Omega$ amplifiers for the ${ }^{234} \mathrm{U}$, the ${ }^{233} \mathrm{U}$ and ${ }^{236} \mathrm{U}$, and the ${ }^{235} \mathrm{U}$ and ${ }^{238} \mathrm{U}$ Faraday cups, respectively. To evaluate the relative gain of the $10^{13} \Omega$ current amplifier prior to sample analyses, we repeated the measurements of the ${ }^{238} \mathrm{U} /{ }^{235} \mathrm{U}$ ratios in a uranium solution with Faraday cups connected to $10^{13}$ and $10^{11} \Omega$ amplifiers. The relative gain of the $10^{13} \Omega$ amplifier was calculated as $\left({ }^{238} \mathrm{U}_{11}{ }^{235} \mathrm{U}_{13}\right) /\left({ }^{238} \mathrm{U}_{11} /\right.$ ${ }^{235} \mathrm{U}_{11}$ ), where the subscript indicates the resistors of the Faraday cup amplifiers. We also developed approaches to correct for the effects of baseline, memory, tailing, hydride, and mass biases. Using the new static measurement method, we repeatedly measured ${ }^{234} \mathrm{U} /{ }^{238} \mathrm{U}$ and ${ }^{238} \mathrm{U} /{ }^{235} \mathrm{U}$ ratios for the international uranium standards HU-1 and SRM950a, as well as for the national uranium standards GBW04412 and GBW04428. Our results (presented with $\pm 2 \sigma$ ) show that the international standards HU-1 $(n=10)$ and SRM950a $(n=10)$ have ${ }^{234} \mathrm{U} /{ }^{238} \mathrm{U}$ atomic ratios of $(54.90 \pm 0.03) \times 10^{-6}$ and $(53.88$ $\pm 0.03) \times 10^{-6}$, and ${ }^{238} \mathrm{U} /{ }^{235} \mathrm{U}$ ratios of $137.765 \pm 0.010$ and $137.844 \pm 0.005$, respectively. On the other hand, the national standards GBW04412 $(n=8)$ and GBW04428 $(n=10)$ have ${ }^{234} \mathrm{U} /{ }^{238} \mathrm{U}$ ratios of $(101.66 \pm 0.04) \times 10^{-6}$ and $(53.86 \pm 0.03) \times 10^{-6}$, and ${ }^{238} \mathrm{U} /{ }^{235} \mathrm{U}$ ratios of $137.786 \pm 0.018$ and $137.995 \pm 0.008$, respectively. Our results are consistent with previously published data and demonstrated high reliability. Our study shows that using the new method we can rapidly measure ${ }^{234} \mathrm{U} /$ ${ }^{238} \mathrm{U}$ and ${ }^{238} \mathrm{U} /{ }^{235} \mathrm{U}$ ratios with precision of $0.3 \%{ }^{-1}-1 \%$ and $0.3 \varepsilon-0.7 \varepsilon$, respectively, consuming $20-30 \mathrm{ng}$ uranium within $\sim 20 \mathrm{~min}$. In addition, our method is flexible: The combination of different amplifiers can be easily modified according to the characteristics of the analyzed samples and the required measurement precision.

Multiple Collector Inductively Coupled Plasma Mass Spectrometry (MC-ICP-MS), $10^{13} \Omega$ amplifier, uranium standard, uranium isotopes

doi: 10.1360/TB-2021-1152 